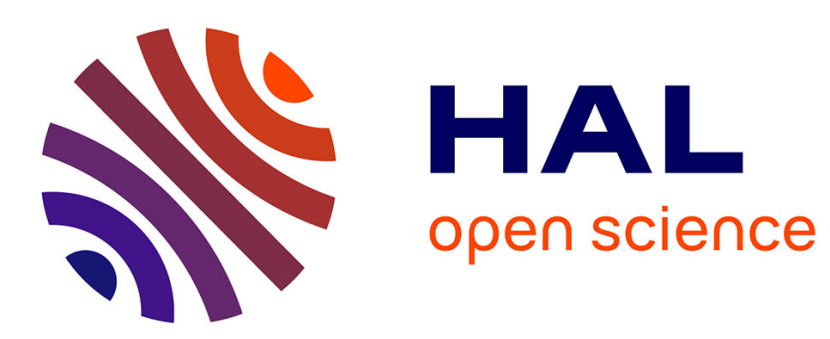

\title{
Weak solutions for a bi-fluid model for a mixture of two compressible non interacting fluids
}

\author{
Antonin Novotny
}

\section{To cite this version:}

Antonin Novotny. Weak solutions for a bi-fluid model for a mixture of two compressible non interacting fluids. Science China Mathematics, 2019, 10.1007/s11425-019-9552-1 . hal-01817433

\section{HAL Id: hal-01817433 \\ https://hal-univ-tln.archives-ouvertes.fr/hal-01817433}

Submitted on 18 Jun 2018

HAL is a multi-disciplinary open access archive for the deposit and dissemination of scientific research documents, whether they are published or not. The documents may come from teaching and research institutions in France or abroad, or from public or private research centers.
L'archive ouverte pluridisciplinaire $\mathbf{H A L}$, est destinée au dépôt et à la diffusion de documents scientifiques de niveau recherche, publiés ou non, émanant des établissements d'enseignement et de recherche français ou étrangers, des laboratoires publics ou privés. 


\title{
Weak solutions for a bi-fluid model for a mixture of two compressible non interacting fluids
}

\author{
Antonin Novotny
}

June 18, 2018

Université de Toulon, IMATH, BP20132, 83957 La Garde, France

\begin{abstract}
We investigate a version of one velocity Baer-Nunziato model with dissipation for the mixture of two compressible fluids with the goal to prove for it the existence of weak solutions for arbitrary large initial data on a large time interval. We transform the one velocity BaerNunziato system to another "more academic" system which possesses the clear "Navier-Stokes structure". We solve the new system by adapting to its structure the Lions approach for solving the (monofluid) compressible Navier-Stokes equations. An extension of the theory of renormalized solutions to the transport equation to more continuity equations with renormalizing functions of several variables is essential in this process. We derive a criterion of almost uniqueness for renormalized solutions to the pure transport equation without classical assumption on the boundedness of the divergence of the transporting velocity. This result does not follow from the DiPerna-Lions transport theory and it is of independent interest. This criterion plays crucial role in the identification of the weak solutions to the original one velocity Baer-Nunziato problem starting from weak solutions of the academic problem. As far as we know, this is the first result on existence of weak solutions for a version of the one velocity bi-fluid system of the Baer-Nunziato type in the mathematical literature.
\end{abstract}

MSC Classification: 76N10, 35Q30

Keywords: bi-fluid system; multifluid system; Baer-Nunziato system; compressible Navier-Stokes equations; transport equation; continuity equation; large data weak solution 


\section{Introduction}

There is no general agreement about the modeling of the mixture of several compressible fluids, and from the general point of view, about two phase flow modeling. One of the acceptable model is the so called two velocity Baer-Nunziato model. The equations of the Baer- Nunziato model with dissipation [4], [8], [18, Section 1] are as follows:

$$
\begin{gathered}
\partial_{t} \alpha_{ \pm}+\mathbf{v}_{I} \cdot \nabla \alpha_{ \pm}=0 \\
\partial_{t}\left(\alpha_{ \pm} \varrho_{ \pm}\right)+\operatorname{div}\left(\alpha_{ \pm} \varrho_{ \pm} \mathbf{u}_{ \pm}\right)=0 \\
\partial_{t}\left(\alpha_{ \pm} \varrho_{ \pm} \mathbf{u}_{ \pm}\right)+\operatorname{div}\left(\alpha_{ \pm} \varrho_{ \pm} \mathbf{u}_{ \pm} \otimes \mathbf{u}_{ \pm}\right)+\nabla\left(\alpha_{ \pm} P_{ \pm}\left(\varrho_{ \pm}\right)\right)-P_{I} \nabla\left(\alpha_{ \pm}\right) \\
=\alpha_{ \pm} \mu_{ \pm}\left(\Delta \mathbf{u}_{ \pm}\right)+\alpha_{ \pm}\left(\mu_{ \pm}+\lambda_{ \pm}\right) \nabla \operatorname{div} \mathbf{u}_{ \pm} \\
0 \leq \alpha_{ \pm} \leq 1, \quad \alpha_{+}+\alpha_{-}=1 .
\end{gathered}
$$

In the above $\left(\alpha_{ \pm}, \alpha_{ \pm} \varrho_{ \pm} \geq 0, \mathbf{u}_{ \pm} \in R^{d}\right)$-concentrations, densities, velocities of the \pm species - are unknown functions of time $t \in I=(0, T), T>0$, and $x \in \Omega \subset R^{d}, d=2,3, \mathrm{n} P_{ \pm}$are two (different) given functions defined on $[0, \infty)$ and $P_{I}, \mathbf{v}_{I}$ are conveniently chosen quantities - they represent pressure and velocity at the interface. In the multifluid modeling, there are many possibilities how the quantities $\mathbf{v}_{I}, P_{I}$ could be chosen, and there is no consensus about this choice.

Our goal in this paper is to prove the existence of weak solutions for the Baer-Nunziato system with dissipation under the following simplifying assumptions:

$$
\begin{gathered}
\mu_{ \pm}:=\mu, \lambda_{ \pm}:=\lambda, \mathbf{v}_{I}=\mathbf{u}_{ \pm}:=\mathbf{u} \\
\alpha P_{ \pm}(s)=\mathscr{P}_{ \pm}\left(f_{ \pm}(\alpha) s\right) \text { for all } \alpha \in(0,1), s \in[0, \infty)
\end{gathered}
$$

with some functions $\mathscr{P}_{ \pm}$defined on $[0, \infty)$ and functions $f_{ \pm}$defined on $(0,1)$.

With this simplifications, the two velocity Baer-Nunziato system reduces to the following system (which we will call the one velocity Baer-Nunziato type system):

$$
\begin{gathered}
\partial_{t} \alpha+(\mathbf{u} \cdot \nabla) \alpha=0, \quad 0 \leq \alpha \leq 1, \\
\partial_{t} \varrho+\operatorname{div}(\varrho \mathbf{u})=0, \\
\partial_{t} z+\operatorname{div}(z \mathbf{u})=0, \\
\partial_{t}((\rho+z) \mathbf{u})+\operatorname{div}((\rho+z) \mathbf{u} \otimes \mathbf{u}) \\
+\nabla P(f(\alpha) \varrho, g(\alpha) z)=\operatorname{div} \mathbb{S}(\nabla \mathbf{u})
\end{gathered}
$$


Here $P:[0, \infty)^{2} \mapsto[0, \infty)$ as well as $f, g:(0,1) \mapsto[0, \infty)$ are given functions, and

$$
\mathbb{S}(\mathbb{Z})=\mu\left(\mathbb{Z}+\mathbb{Z}^{T}\right)+\lambda \operatorname{Tr}(\mathbb{Z}) \mathbb{I}
$$

(II is the identity tensor, $\operatorname{Tr}$ denotes the trace) is the viscous stress tensor. The constant viscosity coefficients satisfy standard physical assumptions, $\mu>0, \lambda+\frac{2}{d} \mu \geq 0$. The system is endowed with initial conditions

$$
\left.\alpha\right|_{t=0}=\alpha_{0},\left.\varrho\right|_{t=0}=\varrho_{0},\left.z\right|_{t=0}=z_{0},\left.(\varrho+z) \mathbf{u}\right|_{t=0}=\left(\varrho_{0}+z_{0}\right) \mathbf{u}_{0},
$$

and no slip boundary conditions,

$$
\left.\mathbf{u}\right|_{\partial \Omega}=\mathbf{0}
$$

Assumption (2) is certainly true in the classical situation of two isentropic gases when

$$
P_{ \pm}(s)=a_{ \pm} s^{\gamma^{ \pm}}, \gamma_{ \pm}>0
$$

indeed, in this case

$P(R, Z)=a_{+} R^{\gamma^{+}}+a_{-} Z^{\gamma^{-}}, f(s):=f_{+}(s)=s^{\frac{1}{\gamma^{+}}}, g(s):=f_{-}(s)=(1-s)^{\frac{1}{\gamma^{-}}}$.

We shall however be able to treat in system (3-7) more general functions $P, f, g$ than those being given by (10).

System (3-6) belongs to the family of multi-fluid models with differential closure, cf. Ishii, Hibiki [20], Drew, Passman [11]. It is not without interest that it can be viewed as a barotropic counterpart of the so called fiveequation bi-fluid model derived in Allaire, Clerc, Kokh [1], [2] by different considerations.

One of the goals of this work is to prove existence of weak solutions for the model (3-7) under quite general assumptions on constitutive functions $P$ and $f, g$ (that include the physical situation evoked in (9) - with however some limitations on admissible values of $\gamma^{ \pm}$which will be specified later). As in [24] (where we have treated another bi-fluid model, with algebraic closure), the proof will be based on the reformulation of the original problem via the change of variables

$$
R:=f(\alpha) \varrho, Z=g(\alpha) z, \Sigma=\varrho+z
$$

and enlarging the new system by another continuity equations for an auxil- 
iary function $\xi$ as follows:

$$
\begin{aligned}
\partial_{t} \xi+\operatorname{div}(\xi \mathbf{u}) & =0, \\
\partial_{t} R+\operatorname{div}(R \mathbf{u}) & =0, \\
\partial_{t} Z+\operatorname{div}(Z \mathbf{u}) & =0, \\
\partial_{t} \Sigma+\operatorname{div}(\Sigma \mathbf{u}) & =0, \\
\partial_{t}(\Sigma \mathbf{u})+\operatorname{div}(\Sigma \mathbf{u} \otimes \mathbf{u})+\nabla P(R, Z) & =\operatorname{div} \mathbb{S}(\nabla \mathbf{u})
\end{aligned}
$$

with boundary and initial conditions

$$
\begin{gathered}
\left.\mathbf{u}\right|_{I \times \partial \Omega}=\mathbf{0} \\
\xi(0, x)=\xi_{0}(x):=\varrho_{0}(x), R(0, x)=R_{0}(x):=f\left(\alpha_{0}\right) \varrho_{0}(x), \\
Z(0, x)=Z_{0}(x):=g\left(\alpha_{0}\right) z_{0}(x), \Sigma(0, x)=\Sigma_{0}(x):=\left(\varrho_{0}+z_{0}\right)(x), \\
\Sigma \mathbf{u}(0, x)=\left(\varrho_{0}+z_{0}\right) \mathbf{u}_{0}(x)=\mathbf{m}_{0}(x)
\end{gathered}
$$

for unknown quintet $(\xi, R, Z, \Sigma, \mathbf{u})$ of functions defined on $Q_{T}$.

This system is of independent interest. In this paper, we will call it an academic bi-fluid system.

In the family of equations (11), the transport equation for $\alpha$ is tacitly hidden in the continuity equations for $\xi$ and $R$ : We anticipate here the fact that (formally), $\xi / R$ verifies transport equation, and that (formally) any sufficiently regular function of $\xi / R$ verifies transport equation as well. This observation will be put on rigorous grounds in Section 3. Another good reason to add to the system the equation for $\xi$ is the construction of solutions via several level of approximations. For the passages from one level to other, it will be important, that the added equation is decoupled from the other equations of the system, and does not modify its Navier-Stokes-like structure.

The statement about the existence of weak solutions for the academic system (11-13) is formulated in Theorem 1 and similar statement about the existence of weak solutions to the one velocity Baer-Nunziato type system $(3-7)$ is available in Theorem 2.

The academic problem (11-13) resembles very much to the academic bi-fluid system treated as an auxiliary problem in [24, Theorem 1] and its analysis will be pretty much inspired by the above reference. The proof of Theorem 1 employs, similarly as [24], the adaptation from mono-fluid to multi-fluid systems of the DiPerna-Lions transport theory [10], and compensated compactness ( leading to the effective viscous flux identity), the tools suggested in the mono-fluid theory by P.L. Lions [21], adapted to the 
multi-fluids in [24] and [25]. The proof of Theorem 2- the passage from the academic system (11-13) to the the Baer-Nunziato system (3-8) needs to employ an original still never exploited observation: It requires, among others, an (almost) uniqueness result for renormalized, bounded solutions of transport equation which seem to differ from what can be deduced from the DiPerna-Lions transport theory [10] and its recent generalizations by Ambrosio, Crippa, De Lellis [3], [7]. It plays crucial role in the identification of quantities $(\alpha, \varrho, z)$ from quantities $(\xi, R, Z, \Sigma)$ in the passage from academic system (11-13) to the final system $(3-8)$. This result is presented in Proposition 5 together with its proof. It is of independent interest.

Theorem 2 is the first rigorous result on existence of weak solutions for a version of the Baer-Nunziato type bi-fluid model.

In general, the results on weak solutions to multi-fluid models are in the mathematical literature in a short supply. It is convenient to quote [25], [22], [24], [5], [6] for a few papers which are relevant to the present work.

The Di-Perna Lions transport theory imposes limitations on adiabatic coefficients $\gamma^{ \pm}$in formula (9) - or an equivalent limitation on growth conditions of $P$ (see the next Section): at least one of them has to be greater or equal than $9 / 5$. In view of the existing mono-fluid theory, existence of weak solutions could be possibly hoped to be achieved if the adiabatic coefficients of constituents were greater than $3 / 2$. This remains however an interesting open problem.

In what follows, the scalar-valued functions will be printed with the usual font, the vector-valued functions will be printed in bold, and the tensorvalued functions with a special font, i.e. $\varrho$ stands for the density, $\mathbf{u}$ for the velocity field and $\mathbb{S}$ for the stress tensor. We use standard notation for the Lebesgue and Sobolev spaces equipped by the standard norms $\|\cdot\|_{L^{p}(\Omega)}$ and $\|\cdot\|_{W^{k, p}(\Omega)}$, respectively. We will sometimes distinguish the scalar-, the vector- and the tensor-valued functions in the notation, i.e. we use $L^{p}(\Omega)$ for scalar quantities, $L^{p}\left(\Omega ; R^{3}\right)$ for vectors and $L^{p}\left(\Omega ; R^{3 \times 3}\right)$ for tensors. The indication of the $R$ or tensor character of the fields (here $; R^{3}$ or $; R^{3 \times 3}$ ) may be omitted, when there is no lack of confusion. The Bochner spaces of integrable functions on $I$ with values in a Banach space $X$ will be denoted $L^{p}(I ; X)$; likewise the spaces of continuous functions on $\bar{I}$ with values in $X$ will be denoted $C(\bar{I} ; X)$. The norms in the Bochner spaces will be denoted $\|\cdot\|_{L^{p}(I ; X)}$ and $\|\cdot\|_{C(\bar{I} ; X)}$, respectively. In most cases, the Banach space $X$ will be either the Lebesgue or the Sobolev space. Finally, we use vector spaces $C_{\text {weak }}(\bar{I} ; X)$ of continuous functions in $\bar{I}$ with respect to weak topology of $X$ (meaning that $f \in C_{\text {weak }}(\bar{I} ; X)$ iff $t \mapsto \mathscr{F}(f(t))$ belongs for any $\mathscr{F} \in X^{*}$ to $C(\bar{I}))$. 
The generic constants will be denoted by $c, \underline{c}, \bar{c}, C, \underline{C}, \bar{C}$ and their value may change even in the same formula or in the same line.

\section{Main results}

\subsection{Definition of weak solutions}

We first explain the notion of the weak solution to problem (11-13) and to problem (3-7):

Definition 1. A quintet $(\xi, \Sigma, R, Z, \mathbf{u})$ is a bounded energy weak solution to problem (11-13), if:

1. $\xi, \Sigma, R, Z \geq 0$ a.e. in $I \times \Omega,(\Sigma, R, Z) \in L^{\infty}\left(I ; L^{\gamma}(\Omega)\right) \cap C_{\text {weak }}\left(\bar{I} ; L^{\gamma}(\Omega)\right)$ with some $\gamma>1, \mathbf{u} \in L^{2}\left(I ; W_{0}^{1,2}\left(\Omega ; R^{3}\right)\right), \Sigma|\mathbf{u}|^{2} \in L^{\infty}\left(I ; L^{1}(\Omega)\right)$, $P(R, Z) \in L^{1}(I \times \Omega), \Sigma \mathbf{u} \in C_{\text {weak }}\left(\bar{I} ; L^{q}(\Omega)\right)$ with some $q>1$.

2. Continuity equations

$$
\int_{0}^{T} \int_{\Omega}\left(r \partial_{t} \psi+r \mathbf{u} \cdot \nabla \psi\right) \mathrm{d} x \mathrm{~d} t+\int_{\Omega} r_{0} \psi(0, \cdot) \mathrm{d} x=0
$$

are satisfied with any $\psi \in C_{c}^{1}([0, T) \times \bar{\Omega})$, where $r$ stands for $\xi, \Sigma, R$, $Z$.

3. Momentum equation

$$
\begin{aligned}
& \int_{0}^{T} \int_{\Omega}\left(\Sigma \mathbf{u} \cdot \partial_{t} \boldsymbol{\varphi}+\Sigma \mathbf{u} \otimes \mathbf{u}: \nabla \boldsymbol{\varphi}+P(R, Z) \operatorname{div} \boldsymbol{\varphi}\right) \mathrm{d} x \mathrm{~d} t \\
= & \int_{0}^{T} \int_{\Omega} \mathbb{S}(\nabla \mathbf{u}): \nabla \boldsymbol{\varphi} \mathrm{d} x \mathrm{~d} t-\int_{\Omega} \mathbf{m}_{0} \cdot \boldsymbol{\varphi}(0, \cdot) \mathrm{d} x
\end{aligned}
$$

holds with any $\varphi \in C_{c}^{1}\left([0, T) \times \Omega ; R^{3}\right)$.

4. Finally, there is function $H \in C^{1}\left((0, \infty)^{2}\right)$ a solution of first order partial differential equation

$$
R \partial_{R} H(R, Z)+Z \partial_{Z} H(R, Z)-H(R, Z)=P(R, Z)
$$

such that energy inequality

$$
\begin{aligned}
& \int_{\Omega}\left(\frac{1}{2} \Sigma|\mathbf{u}|^{2}+H(R, Z)\right)(\tau, \cdot) \mathrm{d} x \\
+ & \int_{0}^{\tau} \int_{\Omega} \mathbb{S}(\nabla \mathbf{u}): \nabla \mathbf{u} \mathrm{d} x \mathrm{~d} t \\
\leq & \int_{\Omega}\left(\frac{1}{2} \Sigma_{0} \mathbf{u}_{0}^{2}+H\left(R_{0}, Z_{0}\right)\right) \mathrm{d} x
\end{aligned}
$$


is satisfied for a.a. $\tau \in(0, T)$.

Definition 2. A quartet $(\alpha, \varrho, z, \mathbf{u})$ is a bounded energy weak solution to problem (3-7), if:

1. $\varrho, z \geq 0$ a.e. in $I \times \Omega$, $(\varrho, z) \in L^{\infty}\left(I ; L^{\gamma}(\Omega)\right) \cap C_{\text {weak }}\left(\bar{I} ; L^{\gamma}(\Omega)\right)$ with some $\gamma>1, \alpha \in L^{\infty}\left(Q_{T}\right) \cap C_{\text {weak }}\left(\bar{I} ; L^{\gamma}(\Omega)\right), 0 \leq \alpha \leq 1$, $\mathbf{u} \in L^{2}\left(I ; W_{0}^{1,2}\left(\Omega ; R^{3}\right)\right),(\varrho+z)|\mathbf{u}|^{2} \in L^{\infty}\left(I ; L^{1}(\Omega)\right), P(f(\alpha) \varrho, g(\alpha) z) \in$ $L^{1}(I \times \Omega),(\varrho+z) \mathbf{u} \in L^{\infty}\left(I ; L^{q}(\Omega)\right) \cap C_{\text {weak }}\left(\bar{I} ; L^{q}(\Omega)\right)$ with some $q>1$.

2. Continuity equations

$$
\int_{0}^{T} \int_{\Omega}\left(r \partial_{t} \psi+r \mathbf{u} \cdot \nabla \psi\right) \mathrm{d} x \mathrm{~d} t+\int_{\Omega} r_{0} \psi(0, \cdot) \mathrm{d} x=0
$$

are satisfied with any $\psi \in C_{c}^{1}([0, T) \times \bar{\Omega})$, where $r$ stands for $\varrho$, $z$.

3. Transport equation

$$
\int_{0}^{T} \int_{\Omega}\left(\alpha \partial_{t} \psi+\alpha \mathbf{u} \cdot \nabla \psi-\psi \alpha \operatorname{div} \mathbf{u}\right) \mathrm{d} x \mathrm{~d} t+\int_{\Omega} \alpha_{0} \psi(0, \cdot) \mathrm{d} x=0
$$

holds with any $\psi \in C_{c}^{1}([0, T) \times \bar{\Omega})$.

4. Momentum equation

$$
\begin{aligned}
& \int_{0}^{T} \int_{\Omega}\left((\varrho+z) \mathbf{u} \cdot \partial_{t} \boldsymbol{\varphi}+(\varrho+z) \mathbf{u} \otimes \mathbf{u}: \nabla \boldsymbol{\varphi}+P(f(\alpha) \varrho, g(\alpha) z) \operatorname{div} \boldsymbol{\varphi}\right) \mathrm{d} x \mathrm{~d} t \\
= & \int_{0}^{T} \int_{\Omega} \mathbb{S}(\nabla \mathbf{u}): \nabla \boldsymbol{\varphi} \mathrm{d} x \mathrm{~d} t-\int_{\Omega}\left(\varrho_{0}+z_{0}\right) \mathbf{u}_{0} \cdot \boldsymbol{\varphi}(0, \cdot) \mathrm{d} x
\end{aligned}
$$

holds with any $\varphi \in C_{c}^{1}\left([0, T) \times \Omega ; R^{3}\right)$.

5. The energy inequality holds

$$
\begin{aligned}
& \int_{\Omega}\left(\frac{1}{2}(\varrho+z)|\mathbf{u}|^{2}+H(f(\alpha) \varrho, g(\alpha) z)\right)(\tau, \cdot) \mathrm{d} x \\
+ & \int_{0}^{\tau} \int_{\Omega} \mathbb{S}(\nabla \mathbf{u}): \nabla \mathbf{u} \mathrm{d} x \mathrm{~d} t \\
\leq & \int_{\Omega}\left(\frac{1}{2}\left(\varrho_{0}+z_{0}\right) \mathbf{u}_{0}^{2}+H\left(f\left(\alpha_{0}\right) \varrho_{0}, g\left(\alpha_{0}\right) z_{0}\right)\right) \mathrm{d} x
\end{aligned}
$$

for a.a. $\tau \in(0, T)$, where $H$ is the same as in (17). 


\subsection{Assumptions}

Motivated by [24, Section 2] we shall gather the hypotheses for Theorems 1 and 2 .

1. Regularity of the domain:

$\Omega$ is a bounded domain of class $C^{2, \nu}, \nu \in(0,1)$.

2. Hypotheses on in initial data:

$$
\begin{gathered}
\left(R_{0}, Z_{0}\right)(x) \in \overline{\mathcal{O}}, 0 \leq \Sigma_{0}(x) \leq \bar{b}\left(R_{0}+Z_{0}\right)(x), \underline{d} R_{0} \leq \xi_{0} \leq \bar{d} R_{0} \\
R_{0} \in L^{\gamma}(\Omega), \gamma \geq \frac{9}{5}, z_{0} \in L^{\beta}(\Omega) \text { if } \beta>\gamma, \Sigma_{0}\left|\mathbf{u}_{0}\right|^{2} \in L^{1}(\Omega) .
\end{gathered}
$$

In the above $\bar{b}>0,0<\underline{d}<\bar{d}$ and

$$
\left.\mathcal{O}:=(R, Z) \in R^{2} \mid \underline{a} R<Z<\bar{a} R\right\}
$$

with some $0<\underline{a}<\bar{a}$.

3. Regularity and growth of the pressure function: Pressure function $P$ : $[0, \infty)^{2} \rightarrow[0, \infty), \mathrm{P}(0,0)=0, P \in C^{1}\left((0, \infty)^{2}\right)$ is such that

$$
\begin{aligned}
& \forall Z \geq 0, \text { function } R \mapsto P(R, Z) \text { is continuous in [0, }), \\
& \quad \forall R \geq 0, \text { function } Z \mapsto P(R, Z) \text { is continuous in }[0, \infty)
\end{aligned}
$$

and there is a number $C \geq 1$ (dependent on $\underline{a}, \bar{a}$ ) such that for all $(R, Z) \in \mathcal{O}$

$$
C^{-1}\left(R^{\gamma}+Z^{\beta}-1\right) \leq P(R, Z) \leq C\left(R^{\gamma}+Z^{\beta}+1\right),
$$

with some $\gamma \geq \frac{9}{5}, \beta>0$.

Moreover,

$$
\left|\partial_{Z} P(R, Z)\right| \leq C\left(R^{-\underline{\Gamma}}+R^{\bar{\Gamma}-1}\right) \text { in } \mathcal{O}
$$

with some $0 \leq \underline{\Gamma}<1$, and with some $0<\bar{\Gamma}<\gamma+\gamma_{B O G}$, where $\gamma_{B O G}=\min \left\{\frac{2}{3} \gamma-1, \frac{\gamma}{2}\right\}$.

Finally, function $R \mapsto P(R, Z)$ is for all $Z>0$ locally Lipschitz on $(0, \infty)$ and function $Z \mapsto \partial_{Z} P(R, Z)$ is for all $R>0$ locally Lipschitz on $(0, \infty)$ with Lipschitz constant

$$
L_{P}(R, Z) \leq C(\underline{r})\left(1+R^{A}\right) \text { for all } \underline{r}>0,(R, Z) \in \mathcal{O}
$$

with some non negative number $A$. Number $C(\underline{r})$ may diverge to $+\infty$ as $\underline{r} \rightarrow 0^{+}$. 
4. Structure of the pressure: It is assumed that

$$
P(R, R s)=\mathscr{P}(R, s)-\mathscr{R}(R, s),
$$

where $[0, \infty) \ni R \mapsto \mathscr{P}(R, s)$ is non decreasing for any $s \in[\underline{a}, \bar{a}]$, and $R \mapsto \mathscr{R}(R, s)$ is for any $s \in[\underline{a}, \bar{a}]$ a non-negative $C^{2}$-function in $[0, \infty)$ uniformly bounded with respect to $s \in[\underline{a}, \bar{a}]$ with compact support uniform with respect to $s \in[\underline{a}, \bar{a}]$. Moreover, if $\gamma=\frac{9}{5}$,

$$
\mathscr{P}(R, s)=\pi(s) R^{\gamma}+p(R, s),
$$

where $[0, \infty) \ni R \mapsto p(R, s)$ is non decreasing for any $s \in[\underline{a}, \bar{a}]$ and $\pi \in L^{\infty}(\underline{a}, \bar{a}), \operatorname{essinf}_{s \in(\underline{a}, \bar{a})} \pi(s) \geq \underline{\pi}>0$. Finally,

$$
\forall R \in(0,1), \sup _{s \in[0, \bar{a}]} P(R, R s) \leq c R^{B} \text { with some } c>0 \text { and } B>0 .
$$

In the above and in the sequel, the following convention is used systematically:

$$
\text { If } 0 \leq Z \leq \bar{a} R \text { then } s=\frac{Z}{R}:=\left\{\begin{array}{c}
\frac{Z}{R} \text { if } \varrho>0, \\
\text { if } R=0 .
\end{array}\right.
$$

\subsection{Main results}

The first main result of the paper deals with the academic system (11-13) and reads

Theorem 1. Under Hypotheses (22-33), problem (11-13) admits at least one weak solution in the sense of Definition 1. Moreover, for all $t \in \bar{I}$, $(R(t, x), Z(t, x)) \in \overline{\mathcal{O}}$ for a.a. $x \in \Omega$, for all $t \in \bar{I}, \underline{d} R(t, x) \leq \xi(t, x) \leq$ $\bar{d} R(t, x)$, for all $t \in \bar{I}, \Sigma(t, x) \leq \bar{b}(R(t, x)+Z(t, x))$ for a.a. $x \in \Omega$, $\xi, Z, R, \Sigma \in C\left(\bar{I} ; L^{1}(\Omega)\right) \cap C_{\text {weak }}\left([0, T) ; L^{\gamma}(\Omega)\right) \cap L^{2}\left(Q_{T}\right), \Sigma \mathbf{u} \in C_{\text {weak }}([0, T)$; $\left.L^{q}\left(\Omega ; R^{3}\right)\right)$ for some $q>1$ and $P(\varrho, Z) \in L^{q}(I \times \Omega)$ for some $q>1$ and $Z \in C_{\text {weak }}\left([0, T) ; L^{\beta}(\Omega)\right)$ if $\beta>\gamma$.

Moreover, energy inequality (17) holds with function

$$
H(R, Z)=R \int_{1}^{R} \frac{P\left(r, \frac{Z}{R} r\right)}{r^{2}} \mathrm{~d} r \text { if }(R, Z) \in(0, \infty)^{2}, H(0,0)=0
$$

which belongs to the space $C^{1}\left((0, \infty)^{2}\right) \cap C(\overline{\mathcal{O}})$.

The second main result of the paper deals with the one velocity BaerNunziato type system (3-8) and reads: 
Theorem 2. Suppose that $f, g \in C^{1}(0,1) \cap C^{1}([\underline{\alpha}, \bar{\alpha}])$ with $0<\underline{\alpha}<\bar{\alpha}<1$ be two strictly monotone and non-vanishing functions on interval $(0,1)$. Let $\gamma \geq 9 / 5, \beta>0$,

$$
\begin{aligned}
& \alpha_{0} \in L^{\infty}(\Omega), 0<\underline{\alpha} \leq \alpha_{0} \leq \bar{\alpha},\left(f\left(\alpha_{0}\right) \varrho_{0}, g\left(\alpha_{0}\right) z_{0}\right)(x) \in \overline{\mathcal{O}} \\
& \varrho_{0} \in L^{\gamma}(\Omega), z_{0} \in L^{\beta}(\Omega) \text { if } \beta>\gamma,\left(\varrho_{0}+z_{0}\right)\left|\mathbf{u}_{0}\right|^{2} \in L^{1}(\Omega) .
\end{aligned}
$$

Suppose that the boundary is sufficintly regular as (22) and the pressure $P$ satisfies hypotheses (26-33). Then the problem (3-8) admits at least one weak solution in the sense of Definition 2. Moreover, for all $t \in \bar{I}$, $(f(\alpha) \varrho(t, x), g(\alpha) z(t, x)) \in \overline{\mathcal{O}}$ for a.a. $x \in \Omega$, for all $t \in \bar{I}, \underline{\alpha} \leq \alpha(t, x) \leq \bar{\alpha}$ for a.a. $x \in \Omega, \alpha, \varrho, z \in C\left(\bar{I} ; L^{1}(\Omega)\right), \varrho, z \in L^{2}\left(Q_{T}\right) z \in C_{\text {weak }}\left(\bar{I} ; L^{\beta}(\Omega)\right)$ if $\beta>\gamma$, and $P(\varrho, Z) \in L^{q}(I \times \Omega)$ with some $q>1$.

\section{Remark 2.1}

1. It is to be noticed that the results of both Theorems 1 and 2 remain valid - after well known necessary modifications in the definition of weak solutions in these cases - in the space periodic setting (if $\Omega$ is a periodic periodic cell) or if we replace the no-slip boundary conditions (8) and (12), respectively, by the Navier conditions

$$
\left.\mathbf{u} \cdot \mathbf{n}\right|_{\partial \Omega}=0,\left[\mu\left(\nabla \mathbf{u}+(\nabla \mathbf{u})^{T}\right)+\lambda I \operatorname{div} \mathbf{u}\right] \mathbf{n} \times\left.\mathbf{n}\right|_{\partial \Omega}=\mathbf{0} .
$$

In the above $\mathbb{I}$ denotes the identity tensor on $R^{3}$ and $\mathbf{n}$ is the outer normal to $\partial \Omega$.

2. Condition (22) on the regularity of the domain $\Omega$ in both Theorems 1 and 2 could be relaxed up to a bounded Lipschitz domain via the technique described in [17].

\section{Preliminaries}

This section contains several preliminary results that will be used later. Most of them is a summary of well known results, except Proposition 5 about the almost uniqueness for the renormalized solutions to the transport equation - whose assumptions are beyond and does not seem to be covered by DiPerna-Lions transport theory [10] and its recent generalizations by Ambrosio, Crippa, De Lellis, see e.g. [3], [7]. 


\subsection{Some consequences of DiPerna-Lions theory of renor- malized solutions to transport equation}

Given $f, v \in L^{1}(I \times \Omega)$ and $\mathbf{u} \in L^{1}\left(0, T ; W^{1,1}(\Omega)\right)$ a function $\varrho \in L^{1}(I \times$ $\Omega ; \overline{\mathscr{R}}), \mathscr{R} \subset R$ an open set, is called a renormalized solution to the general transport equation with transport coefficients $\mathbf{u}, v$ and right hand side $f$ if it satisfies

$$
\partial_{t} \varrho+\mathbf{u} \cdot \nabla \varrho+v \varrho=f \text { in } \mathscr{D}^{\prime}(I \times \Omega)
$$

and

$$
\partial_{t} b(\varrho)+\operatorname{div}(b(\varrho) \mathbf{u})+\left(\varrho b^{\prime}(\varrho)-b(\varrho)\right) \operatorname{div} \mathbf{u}+v b^{\prime}(\varrho) \varrho=f b^{\prime}(\varrho) \text { in } \mathscr{D}^{\prime}(I \times \Omega)
$$

with any

$$
b \in C^{1}([0, \infty)), b^{\prime} \in L^{\infty}((0, \infty)) .
$$

If in (36), $v, f=0$ then we shall call (36) pure transport equation. If $v=\operatorname{div} \mathbf{u}, f=0$ then we call (36) the continuity equation.

The following Proposition resumes the classical consequences of the DiPerna-Lions transport theory [10] applied to two general transport equation involving renormalizing functions of two variables, as formulated in [24, Proposition 5] (compare with [25, Lemma 2.5]).

\section{Proposition 3.}

1. Let functions $\varrho, Z$ satisfy the general transport equation with the same transport coefficients $\mathbf{u}, v$ and with right hand sides $f_{1}$ and $f_{2}$, respectively, in the sense of distributions (cf. equation (36)). Suppose that

$$
\varrho, Z \in L^{2}(I \times \Omega),(\varrho, Z) \in \overline{\mathcal{O}}, \mathbf{u} \in L^{2}\left(I ; W^{1,2}\left(\Omega ; R^{3}\right)\right) v \in L^{2}\left(Q_{T}\right),
$$

where $\mathcal{O}$ is an open set in $R^{2}$. Then for any

$$
b \in C^{1}(\overline{\mathcal{O}}),\left(\partial_{\varrho} b, \partial_{Z} b\right) \in L^{\infty}\left(\mathcal{O} ; R^{2}\right)
$$

the function $b(\varrho, Z)$ verifies the renormalized continuity equation

$$
\begin{aligned}
& \partial_{t} b(\varrho, Z)+\operatorname{div}(b(\varrho, Z) \mathbf{u})+\left(\varrho \partial_{\varrho} b(\varrho, Z)-Z \partial_{Z} b(\varrho, Z)-b(\varrho, Z)\right) \operatorname{div} \mathbf{u}(41) \\
& +(v-\operatorname{divu})\left(\varrho \partial_{\varrho} b(\varrho, Z)+Z \partial_{Z} b(\varrho, Z)\right)=f_{1} \partial_{\varrho} b(\varrho, Z)+f_{2} \partial_{Z} b(\varrho, Z) \\
& \text { in } \mathscr{D}^{\prime}(I \times \Omega) .
\end{aligned}
$$


2. If moreover

$$
\varrho, Z \in L^{\infty}\left(I ; L^{\gamma}(\Omega)\right) \text { with some } \gamma>1, \mathbf{u} \in L^{2}\left(I, W_{0}^{1,2}\left(\Omega ; R^{3}\right)\right),
$$

then we have further:

1. Functions $\varrho, Z, b(\varrho, Z)$ are continuous in time (with values in $L^{1}(\Omega)$ ), namely

$$
\varrho, Z \in C\left(\bar{I} ; L^{1}(\Omega)\right), b(\varrho, Z) \in C\left(\bar{I} ; L^{1}(\Omega)\right) .
$$

2. Both functions $\varrho, Z$ satisfy general transport equation (36) in the time integrated form up to the boundary, namely

$$
\begin{gathered}
\int_{\Omega} r \varphi(\tau, x) \mathrm{d} x-\int_{\Omega} r \varphi(0, x) \mathrm{d} x \\
=\int_{0}^{\tau} \int_{\Omega}\left(r \partial_{t} \varphi+r \mathbf{u} \nabla \varphi-\varphi(v-\operatorname{div} \mathbf{u}) r-f_{i} \varphi\right) \mathrm{d} x
\end{gathered}
$$

for all $\tau \in \bar{I}$ and $\varphi \in C_{c}^{1}(\overline{I \times \Omega})$, where $r$ stands for $\varrho$ if $i=1$ and $Z$ if $i=2$.

3. The renormalized equation (41) is valid in the time integrated form up to the boundary, namely

$$
\begin{gathered}
\int_{\Omega}(b(\varrho, Z) \varphi)(\tau, \cdot) \mathrm{d} x-\int_{\Omega}(b(\varrho, Z) \varphi)(0, x) \mathrm{d} x \\
=\int_{0}^{\tau} \int_{\Omega}\left(b(\varrho, Z) \partial_{t} \varphi+b(\varrho, Z) \mathbf{u} \cdot \nabla \varphi-\left(\varrho \partial_{\varrho} b(\varrho, Z)\right.\right. \\
\left.-Z \partial_{Z} b(\varrho, Z)-b(\varrho, Z)\right) \operatorname{div} \mathbf{u} \varphi-\varphi(v-\operatorname{div} \mathbf{u})\left(\varrho \partial_{\varrho} b(\varrho, Z)\right. \\
\left.\left.+Z \partial_{Z} b(\varrho, Z)\right)+\left(f_{1} \partial_{\varrho} b(\varrho, Z)+f_{2} \partial_{Z} b(\varrho, Z)\right) \varphi\right) \mathrm{d} x \mathrm{~d} t
\end{gathered}
$$

for all $\tau \in \bar{I}$ and $\varphi \in C_{c}^{1}(\overline{I \times \Omega})$.

4. If $\varrho, Z \in L^{\infty}\left(Q_{T}\right)$, then function $b$ can be taken in $C^{1}(\overline{\mathcal{O}})$.

\section{Remark 3.1}

1. Consider now $(\varrho \geq 0, \mathbf{u}, v=0)$ in the regularity class (39-42) verifying continuity (36), and function $b$ of one variable in the class

$$
b \in C([0, \infty)) \cap C^{1}((0, \infty)), b(s) \leq c\left(1+s^{\frac{5}{6} \gamma}\right),
$$




$$
s b^{\prime}-b \in C([0, \infty)), s b^{\prime}(s)-b(s) \leq c\left(1+s^{\gamma / 2}\right),
$$

then

$$
b(\varrho) \in C_{\text {weak }}\left(\bar{I} ; L^{p}(\Omega)\right) \text {, with any } 1 \leq p<6 / 5
$$

and equation (44) still continues to hold in spite of the fact that $b$ is slightly less regular than required in (39).

2. We notice that function $b(\varrho)=\varrho \ln \varrho$ as well as its truncation

$$
\begin{gathered}
L_{k}(\varrho)=\varrho \int_{1}^{\varrho} \frac{T_{k}(z)}{z^{2}} \mathrm{~d} z, T_{k}(z)=k T(Z / k), k>1, \\
T(z)=\left\{\begin{array}{c}
z \text { if } z \in[0,1) \\
2 \text { if } z \geq 3
\end{array}\right\}, T \in C^{\infty}([0, \infty)), \text { concave }
\end{gathered}
$$

verify (45) but fail to satisfy (39). It is this function which is employed in the Feireisl-Lions approach at the very last step of the proof of compactness of the density sequence.

3. We notice that condition $r \in C\left(\bar{I}, L^{1}(\Omega)\right)$ in conjunction with $r \in$ $L^{\infty}\left(I ; L^{p}(\Omega)\right), p>1$ yields by interpolation, $r \in C\left(\bar{I} ; L^{\beta}(\Omega)\right)$ with any $1 \leq \beta<p$. This remark concerns quantities $\varrho, Z, b(\varrho), b(\varrho, Z)$ in Proposition 3 and $s, b(s), \varrho b\left(s_{1}, s_{2}\right)$ in Propositions 4.

\subsection{An almost uniqueness to the solutions of the transport equation}

The next proposition describes passage from two continuity equations to a transport equation, and from transport equation to a continuity equation. It is closely related to Propositions 5 and 6 in [24] whose ideas can be traced back to [22] and [25].

Proposition 4. 1. Suppose that $\varrho, Z, \mathbf{u}$ verify assumptions (39) and (42) with $\mathcal{O}=\left\{(R, Z) \in(0, \infty)^{2} \mid \underline{\mathfrak{a}} R<Z<\overline{\mathfrak{a}} R\right\}$, where $0 \leq \underline{\mathfrak{a}}<\overline{\mathfrak{a}}$ are given numbers. Assume further that couples $(\varrho, \mathbf{u})$ and $(Z, \mathbf{u})$ satisfy transport eaquation (36) with $v=\operatorname{div} \mathbf{u}$ and $f_{1}, f_{2}=0$.

We define for all $t \in \bar{I}, s(t, x)=Z(t, x) / \varrho(t, x)$ in agreement with convention (33).

Then for any $b \in C^{1}[0, \infty), b(s) \in C\left(\bar{I}, L^{1}(\Omega)\right)$ and the couple $(b(s), \mathbf{u})$ satisfies time integrated pure transport equation up to the boundary

$$
\int_{\Omega}(b(s) \varphi)(\tau, \cdot) \mathrm{d} x-\int_{\Omega}(b(s) \varphi)(0, \cdot) \mathrm{d} x
$$




$$
=\int_{0}^{\tau} \int_{\Omega}\left(b(s) \partial_{t} \varphi+b(s) \mathbf{u} \cdot \nabla \varphi-\varphi b(s) \operatorname{div} \mathbf{u}\right) \mathrm{d} x \mathrm{~d} t
$$

for all $\tau \in \bar{I}$ and $\varphi \in C_{c}^{1}(\overline{I \times \Omega})$.

2. Suppose that couples $\left(s_{i}, \mathbf{u}\right), i=1,2$

$$
0 \leq s_{i} \in L^{\infty}\left(Q_{T}\right), \mathbf{u} \in L^{2}\left(I ; W_{0}^{1,2}(\Omega)\right)
$$

satisfy pure transport equation (36) (with $f, v=0)$ and couple $(\varrho, \mathbf{u})$

$$
0 \leq \varrho \in L^{2}\left(Q_{T}\right) \cap L^{\infty}\left(I ; L^{\gamma}(\Omega)\right), \gamma>1
$$

satisfies continuity equation (36) (with $f=0, v=\operatorname{divu}$ ). Then $\varrho, s_{i} \in C\left(\bar{I}, L^{1}(\Omega)\right), \varrho b\left(s_{1}, s_{2}\right)$ with any $b \in C^{1}\left([0, \infty)^{2}\right)$ and the couple $\left(\varrho b\left(s_{1}, s_{2}\right), \mathbf{u}\right)$ verifies time integrated continuity equation up to the boundary

$$
\begin{aligned}
& \int_{\Omega}\left(\varrho b\left(s_{1}, s_{2}\right) \varphi\right)(\tau, \cdot) \mathrm{d} x-\int_{\Omega}\left(\varrho b\left(s_{1}, s_{2}\right) \varphi\right)(0, \cdot) \mathrm{d} x \\
& =\int_{0}^{\tau} \int_{\Omega}\left(\varrho b\left(s_{1}, s_{2}\right) \partial_{t} \varphi+\varrho b\left(s_{1}, s_{2}\right) \mathbf{u} \cdot \nabla \varphi\right) \mathrm{d} x \mathrm{~d} t
\end{aligned}
$$

for all $\tau \in \bar{I}$ and $\varphi \in C_{c}^{1}(\overline{I \times \Omega})$.

Proof. We shall prove the first statement of Proposition 4. We consider a sequence of functions

$$
b_{\delta}:[0, \infty)^{2} \mapsto[0, \infty), b_{\delta}(\varrho, Z)=b\left(\frac{Z}{\varrho+\delta}\right), \delta \in(0,1 / 2) .
$$

Functions $b_{\delta}$ and couples $(\varrho, \mathbf{u})$ and $(Z, \mathbf{u})$ satisfy all assumptions of Proposition 3. Consequently, $b_{\delta}(\varrho, Z) \in C\left(\bar{I}, L^{q}(\Omega)\right), 1 \leq q<\infty$ (by interpolation) and

$$
\partial_{t} b_{\delta}(\varrho, Z)+\operatorname{div}\left(b_{\delta}(\varrho, Z) \mathbf{u}\right)+\left(b^{\prime}\left(\frac{Z}{\varrho+\delta}\right) \frac{\delta \varrho}{(\varrho+\delta)^{2}}-b\left(\frac{Z}{\varrho+\delta}\right)\right) \operatorname{div} \mathbf{u}=0
$$

in $\mathscr{D}^{\prime}\left(Q_{T}\right)$ if $\mathbf{u} \in L^{2}\left(I ; W^{1,2}\left(\Omega ; R^{3}\right)\right)$, or even

$$
\begin{array}{r}
\int_{\Omega}\left(b_{\delta}(\varrho, Z) \varphi\right)(\tau, \cdot) \mathrm{d} x-\int_{\Omega}\left(b_{\delta}(\varrho, Z) \varphi\right)(0, \cdot) \mathrm{d} x=\int_{0}^{\tau} \int_{\Omega}\left(b_{\delta}(\varrho, Z) \partial_{t} \varphi\right. \\
\left.\quad+b_{\delta}(\varrho, Z) \mathbf{u} \cdot \nabla \varphi-\left(b^{\prime}\left(\frac{Z}{\varrho+\delta}\right) \frac{\delta \varrho}{(\varrho+\delta)^{2}}-b\left(\frac{Z}{\varrho+\delta}\right)\right) \operatorname{div} \mathbf{u} \varphi\right) \mathrm{d} x \mathrm{~d} t
\end{array}
$$


if $\mathbf{u} \in L^{2}\left(I ; W_{0}^{1,2}\left(\Omega ; R^{3}\right)\right)$.

We easily verify that for all $t \in \bar{I}$,

$$
\begin{gathered}
\left|b_{\delta}(\varrho, Z)(t, \cdot)\right|+\left|\left(b^{\prime}\left(\frac{Z}{\varrho+\delta}\right) \frac{\delta \varrho}{(\varrho+\delta)^{2}}-b\left(\frac{Z}{\varrho+\delta}\right)\right)(t, \cdot)\right| \leq \bar{a} \text { a.a. in } \Omega \\
\text { for all } t \in \bar{I}, b_{\delta}(\varrho(t, \cdot), Z(t, \cdot)) \rightarrow b(s(t, \cdot)) \text { a.a. in } \Omega
\end{gathered}
$$

and

$$
\text { for all } t \in \bar{I}, b^{\prime}\left(\frac{Z}{\varrho+\delta}\right) \frac{\delta \varrho}{(\varrho+\delta)^{2}} \rightarrow 0 \text { a.a. in } \Omega \text { as } \delta \rightarrow 0 .
$$

We thus obtain via the Lebesgue dominated convergence theorem

$$
\begin{gathered}
\text { for all } \tau \in \bar{I}, \int_{\Omega}\left(b_{\delta}(\varrho, Z) \varphi\right)(\tau, \cdot) \mathrm{d} x \rightarrow \int_{\Omega}(b(s) \varphi)(\tau, \cdot) \mathrm{d} x \\
\text { for all } \tau \in \bar{I}, \int_{0}^{\tau} \int_{\Omega} b_{\delta}(\varrho, Z) \mathbf{u} \cdot \nabla \varphi \mathrm{d} x \mathrm{~d} t \rightarrow \int_{0}^{\tau} \int_{\Omega} b(s) \mathbf{u} \cdot \nabla \varphi \mathrm{d} x \mathrm{~d} t
\end{gathered}
$$

and

$$
\text { for all } \begin{aligned}
& \tau \in \bar{I}, \int_{0}^{\tau} \int_{\Omega}\left(b^{\prime}\left(\frac{Z}{\varrho+\delta}\right) \frac{\delta \varrho}{(\varrho+\delta)^{2}}-b\left(\frac{Z}{\varrho+\delta}\right)\right) \operatorname{div} \mathbf{u} \varphi \mathrm{d} x \mathrm{~d} t \\
& \rightarrow \int_{0}^{\tau} \int_{\Omega} b(s) \operatorname{div} \mathbf{u} \varphi \mathrm{d} x \mathrm{~d} t .
\end{aligned}
$$

We can therefore pass to the limit in equations (50), (51) in order to recover equation (48).

Finally, according to item 3. of Remark 3.1, functions $s, b(s)$ belong to $C\left(\bar{I} ; L^{1}(\Omega)\right)$, and a fortiori, by interpolation, also to $C\left(\bar{I} ; L^{q}(\Omega)\right), 1 \leq q<\infty$. This completes the proof of item 1 . of Proposition 4.

Item 2. of Proposition 4 follows now readily from Proposition 3. This completes the proof of Proposition 4.

The DiPerna Lions transport theory postulates uniqueness of weak solutions to the general transport equation (36) under assumptions $\mathbf{u} \in L^{2}(I$; $\left.W^{1,2}(\Omega)\right), \operatorname{div} \mathbf{u}+v \in L^{\infty}\left(Q_{T}\right)$, cf. [10, Theorem II.2]. The following Proposition shows almost uniqueness to the renormalized solutions to the pure transport equation (i.e. equation (36) with $v=0$ ) without assumption on boundedness of divu. 
Proposition 5. Let $\mathbf{u} \in L^{2}\left(I ; W_{0}^{1,2}\left(\Omega ; R^{3}\right)\right.$. Let $0 \leq s_{i} \in L^{\infty}\left(Q_{T}\right), i=1,2$ be two weak solutions of the pure transport equation (36) (with $v, f=0$ ) in the distributional sense such that $s_{i} \in C\left(\bar{I}, L^{1}(\Omega)\right.$.

If moreover $s_{1}(0, \cdot)=s_{2}(0, \cdot)$ then

$$
\text { for all } \tau \in \bar{I} s_{1}(\tau, \cdot)=s_{2}(\tau, \cdot) \text { for a.a. } x \in\{\varrho(\tau, \cdot)>0\},
$$

where $\varrho$ is any weak solution to the continuity equation (36) (with $v=\operatorname{divu}$, $f=0)$ in the class $0 \leq \varrho \in C\left(\bar{I}, L^{1}(\Omega)\right) \cap L^{2}\left(Q_{T}\right) \cap L^{\infty}\left(I ; L^{p}(\Omega)\right), p>1$.

Proof. According to item 2. of Lemma 4, function $\varrho\left(s_{1}-s_{2}\right)^{2}$ belongs to $C\left(\bar{I} ; L^{1}(\Omega)\right.$ and satisfies continuity equation (49). Taking in this equation the test function $\varphi=1$, we obtain

$$
\text { for all } \tau \in \bar{I} \int_{\Omega} \varrho\left(s_{1}-s_{2}\right)^{2}(\tau, x) \mathrm{d} x=\int_{\Omega} \varrho\left(s_{1}-s_{2}\right)^{2}(0, x) \mathrm{d} x=0 .
$$

This yields the statement.

It is to be noticed that if $\operatorname{div} \mathbf{u} \in L^{1}\left(0, T ; L^{\infty}(\Omega)\right)$ and $0<\underline{c} \leq \varrho_{0}(x)<$ $\bar{c}<\infty$ then $\varrho(t, x)$ in $(52)$ can be constructed by the method of caracteristics which yields for all $t \in \bar{I}, \underline{C}<\varrho(t, x)<\bar{C}$. In this case, Proposition 5 gives uniqueness to renormalized solutions to the transport equation - which is well known classical uniqueness result from [10].

\subsection{A parabolic problem}

The next result follows from the maximal parabolic regularity theory and comparison principle applied to the regularized continuity equation (57), cf. e.g. Denk, Hieber, Prüss [9, Theorem 2.1] and [23, Proposition 7.39].

Proposition 6. Suppose that $\varrho_{0} \in W^{1,2}(\Omega), \mathbf{u} \in L^{\infty}\left(0, T ; W^{1, \infty}\left(\Omega ; R^{3}\right)\right)$, $\left.\mathbf{u}\right|_{(0, T) \times \partial \Omega}=\mathbf{0}$. Then we have:

1. The parabolic problem (57) admits a unique solution in the class

$$
\varrho \in L^{2}\left(I ; W^{2,2}(\Omega)\right) \cap W^{1,2}\left(I ; L^{2}(\Omega)\right) .
$$

2. If moreover $0<\underline{\varrho} \leq \varrho_{0} \leq \bar{\varrho}<\infty$ a.a. in $\Omega$, then there is $0<\underline{c}<\bar{c}<\infty$ dependent on $\bar{\tau}, \underline{\varrho}, \bar{\varrho}$ and $\|\operatorname{div} \mathbf{u}\|_{L^{1}\left(I ; L^{\infty}(\Omega)\right)}$ such that

$$
\text { for all } \tau \in \bar{I}, \underline{c} \leq \varrho(\tau, x) \leq \bar{c} \text { for a.a. } x \in \Omega \text {. }
$$




\section{The academic system: Proof of Theorem 1}

In this section we show how one can prove Theorem 1 referring abundantly to the same proof for similar system presented in [24, Section 4] and putting stress only on eventual differences.

The authors of [24] investigate system (3-8) with $\Sigma=R+Z$ and $\xi=0$ (and $\xi_{0}=0, \Sigma_{0}=R_{0}+Z_{0}$ ). Since the equation for $\xi$ is decoupled from other equations in the system (3-6), the proof of existence of weak solutions to system of equations (3-8) is reduced to the proof presented in the paper $[24$, Section 4] as soon as we show for functions $(\xi, R, Z, \Sigma), \mathbf{u}, \Sigma \mathbf{u}$ the same estimates as those shown for $(R, Z), \mathbf{u},(R+Z) \mathbf{u}$ in [24]. We shall therefore concentrate in the presented proof exclusively to this issue. Paper [24] is needed for the full understanding of all details of this proof.

\subsection{Approximations and estimates}

Following arguments from [24, Section 3], we may suppose, without loss of generality, that

$$
\begin{gathered}
P \in C^{2}\left([0, \infty)^{2}\right), \nabla_{R, Z} P(0,0)=0 \\
0<\underline{R} \leq R_{0}, 0<\underline{\Sigma} \leq \Sigma_{0}, \\
\left(\xi_{0}, R_{0}, Z_{0}, \Sigma_{0}\right) \in C^{3}(\bar{\Omega}),\left.\left(\partial_{\mathbf{n}} \xi_{0}, \partial_{\mathbf{n}} R_{0}, \partial_{\mathbf{n}} Z_{0}, \partial_{\mathbf{n}} \Sigma_{0}\right)\right|_{\partial \Omega}=0 \\
\mathbf{u}_{0} \in C^{3}\left(\bar{\Omega} ; R^{3}\right) \cap W_{0}^{1,2}\left(\Omega ; R^{3}\right) .
\end{gathered}
$$

We take $\delta>0$ and a sufficiently large $B \gg \max \{9 / 2, \gamma, \beta, A\}$ and define

$$
P_{\delta}(\varrho, Z)=P(\varrho, Z)+\delta\left(\varrho^{B}+Z^{B}+\frac{1}{2} \varrho^{2} Z^{B-2}+\frac{1}{2} Z^{2} \varrho^{B-2}\right) .
$$

Next step consists in the parabolic regularization of all continuity equations by adding $\varepsilon \Delta \xi, \varepsilon \Delta R, \varepsilon \Delta Z, \varepsilon \Delta \Sigma$ to the right-hand side of the continuity equations for densities $\xi, \varrho, Z, \Sigma$, respectively, and endowing the new equations with the homogeneous Neumann boundary conditions. It is well known that this regularization must be compensated by adding the term $\varepsilon \nabla \Sigma \cdot \nabla \mathbf{u}$ to the left-hand side of the momentum equation in order to keep in force the energy identity.

Finally we take $\left.\left\{\boldsymbol{\Phi}^{j}\right\}_{j=1}^{\infty} \subset C^{2}\left(\bar{\Omega} ; R^{3}\right)\right) \cap W_{0}^{1,2}\left(\Omega ; R^{3}\right)$ an orthonormal basis in $L^{2}\left(\Omega ; R^{3}\right)$ ) (formed e.g. by eigenfunctions of the Lamé system with homogeneous Dirichlet boundary conditions) and consider for a fixed $N \in \mathbb{N}$ an orthogonal projection of the momentum equation onto the linear hull $\operatorname{LIN}\left\{\boldsymbol{\Phi}^{j}\right\}_{j=1}^{N}$.

To summarize, our approximation looks as follows: 
Definition 3. The quintet ${ }^{1}\left(\xi^{N, \varepsilon, \delta}, R^{N, \varepsilon, \delta}, Z^{N, \varepsilon, \delta}, \Sigma^{N, \varepsilon, \delta}, \mathbf{u}^{N, \varepsilon, \delta}\right)=(\xi, R, Z, \Sigma, \mathbf{u})$ is a solution to our approximate problem, provided $\partial_{t} \varrho, \partial_{t} Z, \partial_{t} \xi, \partial_{t} \Sigma, \nabla^{2} \varrho$, $\nabla^{2} Z, \nabla^{2} \xi, \nabla^{2} \Sigma \in L^{r}(I \times \Omega)$ for some $r \in(1, \infty), \mathbf{u}(t, x)=\sum_{j=1}^{N} c_{j}^{N}(t) \boldsymbol{\Phi}_{j}(x)$ with $c_{j}^{N} \in C^{1}(0, T) \cap C([0, T])$ for $j=1,2, \cdots, N$, the regularized continuity equations

$$
\begin{aligned}
\partial_{t} r+\operatorname{div}(r \mathbf{u}) & =\varepsilon \Delta r, \\
\left.\frac{\partial r}{\partial \mathbf{n}}\right|_{\partial \Omega} & =0 \\
r(0, x) & =r_{0},
\end{aligned}
$$

(where $r$ represents $\xi, R, Z, \Sigma$, according to the case) hold in the a.a. sense, and the Galerkin approximation for the momentum equation

$$
\begin{aligned}
& \int_{0}^{T} \int_{\Omega}\left(\partial_{t}(\Sigma \mathbf{u}) \boldsymbol{\varphi}-\Sigma(\mathbf{u} \otimes \mathbf{u}): \nabla \boldsymbol{\varphi}-P_{\delta}(R, Z) \operatorname{div} \boldsymbol{\varphi}\right) \mathrm{d} x \mathrm{~d} t \\
= & \int_{0}^{T} \int_{\Omega}(\mu \nabla \mathbf{u}: \nabla \boldsymbol{\varphi}+(\mu+\lambda) \operatorname{div} \mathbf{u} \operatorname{div} \boldsymbol{\varphi}-\varepsilon(\nabla \Sigma \cdot \nabla \mathbf{u}) \cdot \boldsymbol{\varphi}) \mathrm{d} x \mathrm{~d} t
\end{aligned}
$$

holds for any $\varphi \in \operatorname{LIN}\{\Phi\}_{j=1}^{N}$, and

$$
\mathbf{u}(0, x)=\mathscr{P}_{N}\left(\mathbf{u}_{0}\right)
$$

with $\mathscr{P}_{N}$ the orthogonal projection onto $\operatorname{LIN}\{\boldsymbol{\Phi}\}_{j=1}^{N}$ in $L^{2}\left(\Omega ; R^{3}\right)$.

\subsubsection{Estimates and limi $N \rightarrow \infty$}

First, applying Proposition 6 to the all regularized continuity equations (57) and to the regularized continuity equation satisfied by the differences $\bar{a} R^{N}-Z^{N}, Z^{N}-\underline{a} R^{N}, \Sigma^{N}-\bar{b}\left(R^{N}+Z^{N}\right)$ and $\bar{d} \xi^{N}-R^{N}$ we easily see that for all $t \in \bar{I}$ and $x \in \Omega$

$$
\begin{gathered}
R^{N}(t, x) \geq C_{1}(\delta, N)>0, Z^{N}(t, x) \geq C_{1}(\delta, N)>0, \\
\underline{d} R^{N}(t, x) \leq \xi^{N}(t, x) \leq \bar{d} R(t, x), 0 \leq \Sigma^{N}(t, x) \leq \bar{b}\left(R^{N}+Z^{N}\right)(t, x) \\
\underline{a} R^{N}(t, x) \leq Z^{N}(t, x) \leq \bar{a} R^{N}(t, x) \leq C_{2}(\delta, N) .
\end{gathered}
$$

Taking in equation (58) test function $\mathbf{u}^{N}$ end employing conveniently equations $(57)_{r=R},(57)_{r=Z},(57)_{r=\Sigma}$ we get after a cumbersome calculation $\frac{\mathrm{d}}{\mathrm{d} t}\left(\frac{1}{2}\left(\left\|\Sigma^{N}\left|\mathbf{u}^{N}\right|^{2}\right\|_{L^{1}(\Omega)}+\int_{\Omega} \mathscr{H}_{\delta}\left(R^{N}, Z^{N}\right) \mathrm{d} x\right)+\varepsilon \int_{\Omega}\left(\frac{\partial^{2} H}{\partial R^{2}}\left(R^{N}, Z^{N}\right)\left|\nabla \varrho^{N}\right|^{2}\right.\right.$

\footnotetext{
${ }^{1}$ We skip the indices $N, \varepsilon$ and $\delta$ in what follows and will use (only one of them) in situations when it will be useful to underline the corresponding limit passage.
} 


$$
\begin{gathered}
\left.+2 \frac{\partial^{2} H}{\partial R \partial Z}\left(R^{N}, Z^{N}\right) \nabla R^{N} \cdot \nabla Z^{N}+\frac{\partial^{2} H}{\partial Z^{2}}\left(R^{N}, Z^{N}\right)\left|\nabla Z^{N}\right|^{2}\right) \mathrm{d} x \\
+\varepsilon \delta B \int_{\Omega}\left(\frac{\partial^{2} h_{\delta}}{\partial R^{2}}\left(R^{N}, Z^{N}\right)\left|\nabla R^{N}\right|^{2}+2 \frac{\partial^{2} h_{\delta}}{\partial R \partial Z}\left(R^{N}, Z^{N}\right) \nabla \varrho^{N} \cdot \nabla Z^{N}\right. \\
\left.+\frac{\partial^{2} h_{\delta}}{\partial Z^{2}}\left(\varrho^{N}, Z^{N}\right)\left|\nabla Z^{N}\right|^{2}\right) \mathrm{d} x+\int_{\Omega}\left(\mu\left|\nabla \mathbf{u}^{N}\right|^{2}+(\mu+\lambda)\left|\operatorname{div} \mathbf{u}^{N}\right|^{2}\right) \mathrm{d} x=0
\end{gathered}
$$

where

$$
\mathscr{H}_{\delta}(\varrho, Z)=H(\varrho, Z)+h_{\delta}(\varrho, Z)
$$

$h_{\delta}(\varrho, Z)=\frac{\delta}{B-1}\left(\varrho^{B}+Z^{B}+\frac{1}{2} \varrho^{2} Z^{B-2}+\frac{1}{2} Z^{2} \varrho^{B-2}\right), H$ is defined in (34). On the other hand, multiplying equation $(27)$ by $\xi, R, Z, \Sigma$ (according to the value of $r$ ) and integrating over $\Omega$ we obtain the identities

$$
\frac{1}{2} \frac{\mathrm{d}}{\mathrm{d} t}\|r\|_{L^{2}(\Omega)}^{2}+\varepsilon\|\nabla r\|_{L^{2}(\Omega)}^{2}=-\frac{1}{2} \int_{\Omega} r^{2} \operatorname{div} \mathbf{u}^{N} \mathrm{~d} x
$$

where $r$ stands for $\xi, R, Z, \Sigma$.

Using equations (61) (together with the structure of $H$ inherited from $P$ through explicit formula (34) - here, the additional assumption (54) is important) and (62) as well as the domination of $\xi$ by $R, \Sigma$ by $R, Z$, and $Z$ by $R$ established in (60), we end finally with

$$
\begin{gathered}
\left\|\xi^{N}, \varrho^{N}, Z^{N}, \Sigma^{N}\right\|_{L^{\infty}\left(I ; L^{B}(\Omega)\right)}+\left\|\Sigma^{N}\left|\mathbf{u}^{N}\right|^{2}\right\|_{L^{\infty}\left(I ; L^{1}(\Omega)\right)} \\
+\left\|\mathbf{u}^{N}\right\|_{L^{2}\left(I ; W^{1,2}(\Omega)\right)}+\varepsilon\left\|\nabla \varrho^{N}, \nabla Z^{N}\right\|_{L^{2}\left(I ; L^{2}(\Omega)\right)}^{2} \leq C
\end{gathered}
$$

where the constant $C$ is independent of $N$ and $\varepsilon$ (but blows up when $\delta \rightarrow$ $\left.0^{+}\right)$.

At this stage, we have for quantities $\xi^{N}$ and $\Sigma^{N}$ the same estimates as for quantities $Z^{N}, R^{N}$. We can therefore pass to the limit $N \rightarrow \infty$ exactly in the same way as it is done in [24, Section 4.2]. Denoting by by $(\xi, R, Z, \Sigma)$ the corresponding (weak) limits (as $N \rightarrow \infty$ ) we may infer that they satisfy regularized continuity equations (57), momentum equation

$$
\begin{gathered}
\int_{0}^{T} \int_{\Omega}\left(\Sigma \mathbf{u} \cdot \partial_{t} \boldsymbol{\varphi}+\Sigma \mathbf{u} \otimes \mathbf{u}: \nabla \boldsymbol{\varphi}+P_{\delta}(R, Z) \operatorname{div} \boldsymbol{\varphi}\right) \mathrm{d} x \mathrm{~d} t \\
=\int_{0}^{T} \int_{\Omega}(\mu \nabla \mathbf{u}: \nabla \boldsymbol{\varphi}+(\mu+\lambda) \operatorname{div} \mathbf{u} \operatorname{div} \boldsymbol{\varphi}-\varepsilon \nabla \Sigma \cdot \nabla \mathbf{u}) \mathrm{d} x \mathrm{~d} t-\int_{\Omega} \mathbf{m}_{0} \cdot \boldsymbol{\varphi}(0, \cdot) \mathrm{d} x
\end{gathered}
$$

with any $\varphi$ in $C_{c}^{1}([0, T) \times \bar{\Omega})$, and the energy identity (61), written as

$$
\left(\frac{1}{2}\left\|\Sigma|\mathbf{u}|^{2}(t)\right\|_{L^{1}(\Omega)}+\int_{\Omega} \mathscr{H}_{\delta}(\varrho, Z)(t) \mathrm{d} x\right)
$$




$$
\begin{gathered}
+\int_{0}^{t} \int_{\Omega}\left(\mu|\nabla \mathbf{u}|^{2}+(\mu+\lambda)|\operatorname{div} \mathbf{u}|^{2}\right) \mathrm{d} x \mathrm{~d} \tau+\varepsilon \int_{0}^{t} \int_{\Omega}\left(\frac{\partial^{2} \mathscr{H}_{\delta}}{\partial \varrho^{2}}(\varrho, Z)|\nabla \varrho|^{2}\right. \\
\left.+2 \frac{\partial^{2} \mathscr{H}_{\delta}}{\partial \varrho \partial Z}(\varrho, Z) \nabla \varrho \cdot \nabla Z+\frac{\partial^{2} \mathscr{H}_{\delta}}{\partial Z^{2}}(\varrho, Z)|\nabla Z|^{2}\right) \mathrm{d} x \mathrm{~d} \tau \\
\leq \int_{\Omega}\left(\frac{1}{2} \Sigma_{0} \mathbf{u}_{0}^{2}+\mathscr{H}_{\delta}\left(\varrho_{0}, Z_{0}\right)\right) \mathrm{d} x
\end{gathered}
$$

for a.a. $t \in(0, T)$.

\subsubsection{Estimates and limit $\varepsilon \rightarrow 0$}

We shall list the available estimates for the quintet $\left(\xi_{\varepsilon}, R_{\varepsilon}, Z_{\varepsilon}, \Sigma_{\varepsilon}, \mathbf{u}_{\varepsilon}\right)=$ $(\xi, R, Z, \Sigma, \mathbf{u})$. Inequalities (60) give in the limit

$$
\begin{aligned}
& \forall t \in \bar{I}, R_{\varepsilon}(t, x) \geq 0, Z_{\varepsilon}(t, x) \geq 0, \underline{d} R_{\varepsilon}(t, x) \leq \xi_{\varepsilon}(t, x) \leq \bar{d} R_{\varepsilon}(t, x), \\
& \quad \underline{a} R_{\varepsilon}(t, x) \leq Z_{\varepsilon}(t, x) \leq \bar{a} R_{\varepsilon}(t, x), 0 \leq \Sigma_{\varepsilon}(t, x) \leq \bar{b}\left(R_{\varepsilon}+Z_{\varepsilon}\right)(t, x)
\end{aligned}
$$

for a. a. $x \in \Omega$. Employing the properties $\mathscr{H}_{\delta}$ induced by explicit formula (34) for $H$ (here, still, for the last time, assumption (54) is important) and (62), we derive from the energy inequality (66),

$$
\begin{gathered}
\left\|\varrho_{\varepsilon}\left|\mathbf{u}_{\varepsilon}\right|^{2}\right\|_{L^{\infty}\left(I ; L^{1}(\Omega)\right)}+\left\|\left(\xi_{\varepsilon}, R_{\varepsilon}, Z_{\varepsilon}, \Sigma_{\varepsilon}\right)\right\|_{L^{\infty}\left(I ; L^{B}(\Omega)\right)} \\
+\sqrt{\varepsilon}\left\|\nabla \xi_{\varepsilon}, \nabla \varrho_{\varepsilon}, \nabla Z_{\varepsilon}, \nabla \Sigma_{\varepsilon}\right\|_{L^{2}\left(Q_{T}\right)}+\left\|\mathbf{u}_{\varepsilon}\right\|_{L^{2}\left(I ; W^{1,2}(\Omega)\right)} \leq C,
\end{gathered}
$$

where we have used also the domination estimates (67).

These are exactly the same starting estimates which were needed for the passage $\varepsilon \rightarrow 0$ in [24, Section 4.3]. We can therefore repeat line by line the reasoning of that paper - including improved estimates of density via the Bogovskii-type test functions, including arguments yielding convergence of $\int_{\Omega} R_{\varepsilon}\left(s_{\varepsilon}-s\right)^{2} \mathrm{~d} x$ to 0 (with $s_{\varepsilon}=Z_{\varepsilon} / R_{\varepsilon}$ and $s=Z / R$ where $Z$ and $R$ are weak limits of the sequences $Z_{\varepsilon}$ and $R_{\varepsilon}$ ), including effective viscous flux identity and including final reasoning using renormalized continuity equation with renormalizing function $R \log R$.

Denoting by by $\left(\xi_{\delta}, R_{\delta}, Z_{\delta}, \Sigma_{\delta}, \mathbf{u}_{\delta}\right)=(\xi, R, Z, \Sigma, \mathbf{u})$ the corresponding (weak) limit (as $\varepsilon \rightarrow 0+$ ) we may infer that it satisfies the weak formulations of the continuity equations, see (14), the weak formulation of the momentum equation (15) with $P$ replaced by $P_{\delta}$ and energy inequality $(66)_{\xi_{\delta}, R_{\delta}, Z_{\delta}, \Sigma_{\delta}, \mathbf{u}_{\delta}}$. 


\subsubsection{Estimates and limit $\delta \rightarrow 0$}

The final step in the prove of Theorem 1 is the limit $\delta \rightarrow 0$. As in the previous Section, we deduce from (67) the following estimates

$$
\begin{gathered}
R_{\delta}(t, x) \geq 0, Z_{\delta}(t, x) \geq 0, \underline{d} R_{\delta}(t, x) \leq \xi_{\delta}(t, x) \leq \bar{d} R_{\delta}(t, x), \\
\underline{a} R_{\delta}(t, x) \leq Z_{\delta}(t, x) \leq \bar{a} R_{\delta}(t, x), 0 \leq \Sigma_{\delta}(t, x) \leq \bar{b}\left(R_{\delta}+Z_{\delta}\right)(t, x)
\end{gathered}
$$

for a. a. $x \in \Omega$. Taking into account (34) and (62) together with assumption (27), we deduce from energy inequality $(66)_{\xi_{\delta}, R_{\delta}, Z_{\delta}, \Sigma_{\delta}, \mathbf{u}_{\delta}}$,

$$
\begin{gathered}
\left\|\varrho_{\delta}\left|\mathbf{u}_{\delta}\right|^{2}\right\|_{L^{\infty}\left(I ; L^{1}(\Omega)\right)}+\delta^{1 / B}\left\|\left(\xi_{\delta}, R_{\delta}, Z_{\delta}, \Sigma_{\delta}\right)\right\|_{L^{\infty}\left(I ; L^{B}(\Omega)\right)} \\
+\left\|\left(\xi_{\delta}, R_{\delta}, Z_{\delta}, \Sigma_{\delta}\right)\right\|_{L^{\infty}\left(I ; L^{\gamma}(\Omega)\right)}+\left\|\mathbf{u}_{\delta}\right\|_{L^{2}\left(I ; W^{1,2}(\Omega)\right)} \leq C .
\end{gathered}
$$

Testing the momentum equation $(15)_{P=P_{\delta}}$ with the Bogovskii solution of the divergence equation $\operatorname{div} \varphi=\varrho^{\eta}-\frac{1}{|\Omega|} \int_{\Omega} \varrho^{\eta} \mathrm{d} x,\left.\varphi\right|_{\partial \Omega}=0$ (with convenient number $\eta>0$ ), we arrive to improve the estimate of density up to

$$
\left\|R_{\delta}\right\|_{L^{\bar{\gamma}(I)}} \leq C, \text { where } \bar{\gamma}=\gamma+\min \left\{\gamma / 2, \frac{2}{3} \gamma-1\right\} .
$$

This estimate is inherited also by $\xi_{\delta}, Z_{\delta}, \Sigma_{\delta}$ by virtue of the domination estimate (69). We notice that if $\gamma \geq 9 / 5$ then $\bar{\gamma} \geq 2$. This observation is crucial: it guarantees that the quantities $\xi, R, Z, \Sigma$ are square integrable, which is one of the most essential assumptions to let work the theory of renormalized solutions reported in Section 3. These are exactly the same starting estimates which were needed for the passage $\delta \rightarrow 0$ in $[24$, Section 4.4]. The reader can now repeat line by line the (detailed) reasoning available in that paper. Theorem 1 is proved.

\section{One velocity Baer-Nunziato system: Proof of Theorem 2}

We set

$$
F(\alpha)=\frac{1}{f(\alpha)}, G(\alpha)=\frac{1}{g(\alpha)},
$$

where clearly $F, G$ are strictly monotone non vanishing functions on interval $(0,1)$, and denote

$$
\underline{F}=\min \{F(\underline{\alpha}), F(\bar{\alpha})\}, \bar{F}=\max \{F(\underline{\alpha}), F(\bar{\alpha})\} .
$$


Similarly, we define numbers $\underline{G}, \bar{G}$ as above replacing function $F$ by $G$. Then, in particular, $F:[\underline{\alpha}, \bar{\alpha}] \rightarrow[\underline{F}, \bar{F}]$ is a $C^{1}$-diffeomorphism.

We will use Theorem 1 with initial conditions

$$
\xi_{0}=\varrho_{0}, R_{0}=f\left(\alpha_{0}\right) \varrho_{0}, Z_{0}=g\left(\alpha_{0}\right) z_{0}, \Sigma_{0}=\varrho_{0}+z_{0}, \mathbf{u}_{0} .
$$

If $\left(\alpha_{0}, \varrho_{0}, z_{0}\right)$ satisfies conditions (35) then $\left(\xi_{0}, R_{0}, Z_{0}, \Sigma_{0}\right)$ verifies $(23)$ with $\underline{a}, \bar{a}$ for the relation between $R_{0}, Z_{0}, \underline{d}=\underline{F}, \bar{d}=\bar{F}$ for the relation between $\xi_{0}, R_{0}$ and $\bar{b}=\max \{\bar{F}, \bar{G}\}$ for the relation between $\Sigma_{0}$ and $R_{0}+Z_{0}$.

Theorem 1 guarantees existence of a weak solution $(\xi, R, Z, \Sigma, \mathbf{u})$ in the corresponding regularity class described in that theorem. Therefore, couples $(\xi, \mathbf{u}),(R, \mathbf{u})$, verify assumptions of Proposition 4 with $\underline{\mathfrak{a}}=\underline{F}, \overline{\mathfrak{a}}=\bar{F}$.

We now set

$$
\alpha=F^{-1}(\xi / R), \varrho=F(\alpha) R, z=G(\alpha) Z .
$$

Clearly, according to item 1 . of Proposition $4, \xi / R$ and $\alpha$ satisfy pure transport equation (19) with boundary conditions $\xi / R(0)=\xi_{0} / R_{0}=F\left(\alpha_{0}\right)$ and $\alpha(0)=\alpha_{0}$, respectively. Now, according to item 2. of Proposition 4, the couple $(\varrho, \mathbf{u})$ satisfies the continuity equation (18) with initial condition $\varrho(0)=F\left(\alpha_{0}\right) R_{0}=\varrho_{0}$. Likewise, the couple $(z, \mathbf{u})$ satisfies the continuity equation (18) with initial condition $z(0)=G\left(\alpha_{0}\right) Z_{0}=z_{0}$.

The proof will be finished if we show that for all $t \in \bar{I}, \Sigma(t, \cdot)=\varrho(t, \cdot)+$ $z(t, \cdot)$ a.e. in $\Omega$.

To this end we observe that again according to item 1. of Proposition 4 the quantity $z / \varrho$ together with $\mathbf{u}$ satisfies transport equation (19) with initial condition $z_{0} / \varrho_{0}$, and consequently the quantity $T=\Sigma / R$ together with $\mathbf{u}$ satisfies transport equation (19) with initial datum $T(0)=F\left(\alpha_{0}\right)+F\left(\alpha_{0}\right) \frac{z_{0}}{\varrho_{0}}$. On the other hand, by the same token the quantity $S=z / R+\varrho / R$ verifies transport equation (19) with the same initial datum as the quantity $T$. We thus conclude that for all $t \in \bar{I}, R T(t, \cdot)=R S(t, \cdot)$ a.e. in $\Omega$, by virtue of Proposition 5. In the other words for all $t \in \bar{I}, \Sigma(t, \cdot)=\varrho(t, \cdot)+z(t, \cdot)$ a.e. in $\Omega$. This completes the proof of Theorem 2 .

\section{References}

[1] G. Allaire, S. Clerc, S. Kokh A five-equation model for the numerical simulation of interfaces in two-phase flows CRAS, Série I 331: 10171022, 2000 .

[2] A five-equation model for the numerical simulation of interfaces between compressible fluids J. Comp. Phys., 181: 577-616, 2002 
[3] Ambrosio, L., Crippa, G. Existence, uniqueness, stability and differentiability properties of the flow associated to weakly differentiable vector fields. Transport equations and multi-D hyperbolic conservation laws, 357, Lect. Notes Unione Mat. Ital., 5, Springer, Berlin, 2008.

[4] M. R. Baer, J. W. Nunziato. A two-phase mixture theory for the deflagration-to-detonation transition (DDT) in reactive granular materials. International journal of multiphase flow 12(6):861-889, 1986.

[5] D. Bresch, B. Desjardins, J.M. Ghidaglia, E. Grenier, M. Hilliairet. Multifluid models including compressible fluids. Handbook of Mathematical Analysis in Mechanics of Viscous Fluids, Eds. Y. Giga et A. Novotný (2018), pp. 52.

[6] D. Bresch, P.B. Mucha, E. Zatorska. Finite-Energy Solutions for Compressible Two-Fluid Stokes System. arXiv: 1709.03922.

[7] G. Crippa, C. De Lellis. Existence, Uniqueness, Stability and Differentiability Properties of the Flow Associated to Weakly Differentiable Vector Fields. J. reine angew. Math. 616, 15-46, 2008

[8] S. Dallet A comparative study of numerical schemes for the BaerNunziato model Preprint: hal-01412148, 2016

[9] R. Denk, M. Hieber, and J. Prüss. Optimal $L p-L q$-estimates for parabolic boundary value problems with inhomogeneous data. Math. Z. 257, 193-224, 2007.

[10] R.J. DiPerna, P.-L. Lions. Ordinary differential equations, transport theory and Sobolev spaces. Invent. Math. 98, 511-547, 1989.

[11] D. Drew, S.L. Passman. Theory of multicomponent fluids. Springer Verlag. Applied Math Sciences, 135 (1999).

[12] E. Feireisl. Compressible Navier-Stokes equations with a non-monotone pressure law. J. Differential Equations 184, 97-108, 2002.

[13] E. Feireisl. Dynamics of viscous compressible fluids. Oxford University Press. Oxford Lecture Series in Mathematics and its Applications, 26, (2004).

[14] E. Feireisl, R. Klein, A. Novotný, E. Zatorska. On singular limits arising in the scale analysis of stratified fluid flows. Math. Models Methods Appl. Sci. 26, 419-443, 2016+. 
[15] E. Feireisl, A. Novotný. Singular limits in thermodynamics of viscous fluids. Birkhäuser Verlag. Advances in Mathematical Fluid Mechanics, (2009).

[16] E. Feireisl, A. Novotný, H. Petzeltová. On the existence of globally defined weak solutions to the Navier-Stokes equations of compressible isentropic fluids. J. Math. Fluid Mech. 3, 358-392, 2001.

[17] E. Feireisl, A. Novotný, H. Petzeltová. On the domain dependence of solutions to the compressible Navier-Stokes equations of a barotropic fluid. Math. Methods Appl. Sci. 25, 1045-1073, 2002.

[18] V. Guillemaud. Modélisation et simulation numérique des écoulements diphasiques par une approche bifuide à deux pressions. $\mathrm{PhD}$ thesis, Université de Provence-Aix-Marseille I, 2007. https://tel. archives-ouvertes.fr/

[19] G.P. Galdi. An introduction to the mathematical theory of the NavierStokes equations. Steady-state problems. Second edition. Springer Verlag. Springer Monographs in Mathematics, (2011).

[20] M. Ishii, T. Hibiki. Thermo-fluid dynamics of two-phase flow. Springer Verlag (2006).

[21] P.-L. Lions. Mathematical topics in fluid mechanics. Vol. 2. Compressible models. Oxford Science Publications. Oxford Lecture Series in Mathematics and its Applications 10, (1998).

[22] D. Maltese, M. Michálek, P.B. Mucha, A. Novotný, M. Pokorný, E. Zatorska. Existence of weak solutions for compressible Navier-Stokes equations with entropy transport. J. Differential Equations 261, 44484485, 2016.

[23] A. Novotný, I. Straškraba. Introduction to the mathematical theory of compressible flow. Oxford University Press. Oxford Lecture Series in Mathematics and its Applications 27, (2004).

[24] A. Novotny, M. Pokorny. Weak solutions for some compressible multicomponent fluid models https://arxiv.org/pdf/1802.00798.pdf

[25] A. Vasseur, H. Wen, C. Yu. Global weak solution to the viscous twofluid model with finite energy. arXiv: 1704.07354. 